\title{
Nanoscale mobility mapping in semiconducting polymer films
}

\author{
A. Alekseev ${ }^{1,2^{*}}$, A. Yedrissov ${ }^{3}$, G. J. Hedley ${ }^{4}$, O. Ibraikulov ${ }^{5}$, T. Heiser ${ }^{5}$, I.D.W. Samuel ${ }^{6}$, S. Kharintsev ${ }^{2}$ \\ ${ }^{1}$ National Research University “MIET”, Moscow, 124498, Russia \\ ${ }^{2}$ Kazan Federal University, Kazan, 420008, Russia \\ ${ }^{3}$ NLA, Nazarbayev University, 010000 Nur-Sultan, Kazakhstan \\ ${ }^{4}$ University of Glasgow, Glasgow G12 8QQ, UK \\ ${ }^{5}$ Strasbourg University, 67081 Strasbourg, France \\ ${ }^{6}$ University of St Andrews, St Andrews KY16 9SS, UK \\ *e-mail: alalrus@gmail.com
}

\begin{abstract}
Local electric properties of thin films of the polymer PTB7 are studied by conductive atomic force microscopy (C-AFM). Non-uniform nanoscale current distribution in the neat PTB7 film is revealed and connected with the existence of ordered PTB7 crystallites. The shape of local $I-V$ curves is explained by the presence of space charge limited current. We modify an existing semi-empirical model for estimation of the nanoscale hole mobility from our experimental C-AFM measurements. The procedure of nanoscale charge mobility estimation was described and applied to the PTB7 films. The calculated average C-AFM hole mobility is in good agreement with macroscopic values reported for this material. Mapping of nanoscale hole mobility was achieved using the described procedure. Local mobility values, influenced by nanoscale structure, vary more than two times in value and have a root-mean-square value $0.22 \times 10^{-8}$ $\mathrm{m}^{2} /(\mathrm{Vs})$, which is almost $20 \%$ from average hole mobility.
\end{abstract}

\section{Keywords}

Charge carrier mobility; current distribution; organic semiconductor; space charge limited current; conductive AFM.

\section{Introduction}

The remarkable progress in development of bulk heterojunction polymer solar cells during last decade enable to routinely achieve more than $10 \%$ power conversion efficiencies (PCE) by single junction devices and recently achieved $16 \%$ power conversion efficiency[1-3]. The most significant driving force of such progress is the synthesis of new materials. One of the most important achievements of the last decade was the introduction of a new benzodithiophene based polymer electron donor with abbreviation PTB7 [4]. The improved processing conditions and device architecture helped to achieve 9.2\% [5] and later 10.02\% PCE [6] on solar cells with PTB7:PC ${ }_{71} \mathrm{BM}$ active layers. Thus, the PTB7:PC ${ }_{71} \mathrm{BM}$ blend has seen significant investigation since its first introduction and the structure-property relationship of this blend have been extensively examined by different methods [7-13]. One of the important parameters in organic semiconductors, drastically influencing device performance, is the charge carrier mobility, which can be determined by different methods, in particular, by measurements of the space charge limited current (SCLC) $[4,12,14-18]$. Influence of the hole mobility on the performance of PTB7:PC ${ }_{71} B M$ solar cells was established in [12]. Beside the structure of the bulk heterojunction, characterization of electrical properties of organic semiconductors at the nanoscale is one of the most promising directions in trying to understand the physics of organic electronic devices and improving their performance. The use of small electrodes to form local contacts is required for such nanoscale measurements. Thus, atomic force microscopy (AFM) equipped with a conductive probe (conductive-AFM or C-AFM) is a convenient tool for probing local electrical properties of materials. Previously, C-AFM was employed for nanoscale electrical characterization of different organic materials and blends [10,11,19-25]. Visualization of the donor and acceptor phases in bulk heterojunction polymer solar cell as well as a local (photo)conductivity study were performed using this technique. Different electronic properties of components in bulk heterojunction layers 
allowed different components in blends to be successful distinguished. Additionally, the local $I-V$ curve measurements are helpful tool for analysis of the local electrical properties, such as charge carrier mobility, quantum efficiency, local open circuit voltage and short circuit current [11, 20-23]. Quantitative analysis of local $I-V$ curves can be very efficient tool for characterization of the local electrical properties of organic solar cells. However, complicated geometry of the electrodes in C-AFM makes any quantitative analysis much more difficult than characterization of the device with flat electrodes. For example, it is known that the standard Mott-Gurney law is not applicable for C-AFM geometry when space charge limited current is used for charge mobility estimation [22]. Values of local mobility in OPV materials were more than an order of magnitude higher than macroscopic data obtained by standard macroscopic SCLC method when Mott-Gurney law was used. The first semi-empirical model for local mobility measurements by C-AFM was introduced by O. Reid et al. [22] and it was applied to well-known standard OPV materials such as P3HT and MDMO-PPV. The measurements of the local charge carrier mobility based on the proposed method showed good agreement with macroscopic SCLC data.

Here, we use C-AFM for analysis of the local conductivity in a neat PTB7 film and use a modified semiempirical model of SCLC [22] for calculation of the nanoscale mobility and its variations caused by film structure. A distribution of hole mobilities over the film surface is mapped using local $I-V$ data, and statistical data for the local electrical parameters are obtained.

\section{Materials and methods}

Neat PTB7 films were produced by spin-coating from solution in chlorobenzene on glass/ITO/PEDOT:PSS as described elsewhere $[10,11]$. In total 8 samples of the neat PTB7 films were prepared by using various rotation speed, resulting in 8 different film thicknesses in the range $50-120 \mathrm{~nm}$ as determined by AFM. The thicknesses of films were measured near a deliberate scratch by AFM operating in tapping mode. Noncontact probes NSG10 (NT-MDT) were utilized. The result of scanning is shown in Fig. 1a, where three levels of surface are seen. The thickness of film was determined by using statistics as distance between peaks, which correspond to three levels of surface: ITO, PEDOT:PSS and PTB7 (Fig. 1b). The ITO is at bottom of scratched area.
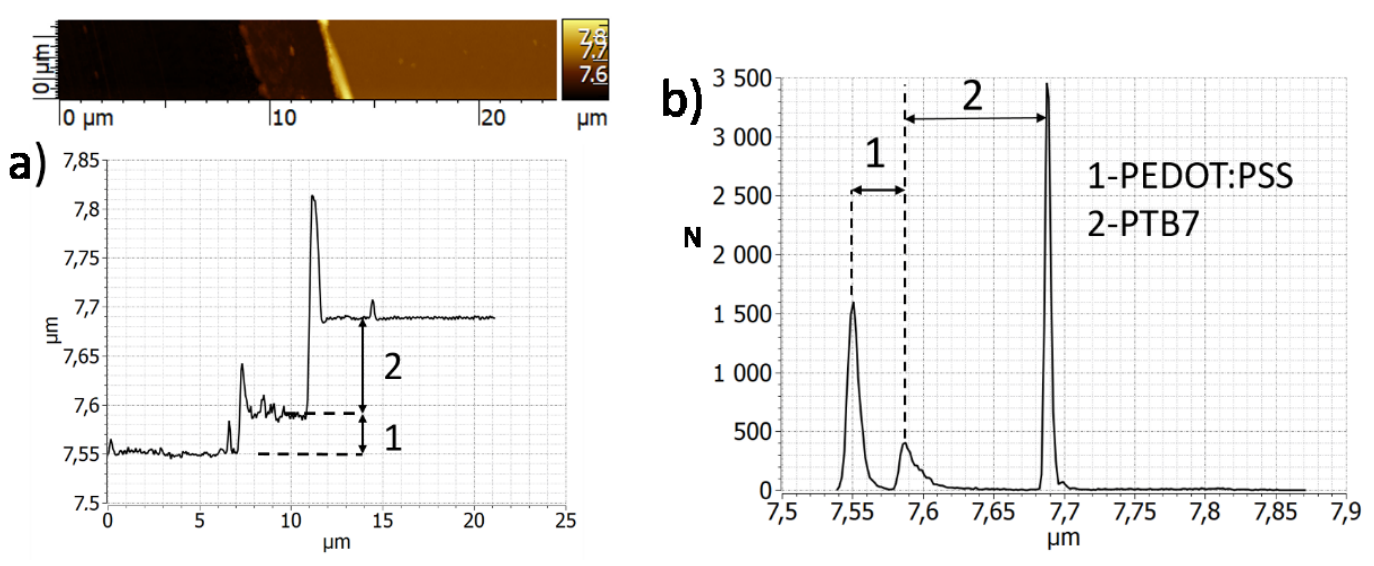

Fig. 1. a) Topography of the scratched area and cross-section of topography; b) determination of film thicknesses by using distribution of the number of pixels over $z$-coordinate.

C-AFM measurements were performed by AFM Smart1000 (Horiba) in contact regime. The Au-coated probes CSG10/Au were utilized for C-AFM. An alternating mode of current measurements implemented in software was employed, which implies the lift of the probe from surface when movement from point to point is executed in order to reduce sample damage. The average tip-sample force was estimated from several force-distance measurements for each experiment. The cantilever force constant was calculated by using the Sader method embedded into AFM software [26]. The tip-sample area was estimated corresponding to Hertzian contact theory with modulus elasticity of PTB7 of $0.44 \mathrm{GPa}$, which was taken from recently published data [13]. Force-distance curves were obtained for each set of local $I-V$ measurements and used for estimation of the tip-sample force (see Supplementary Information for details). For all calculations the tip was treated as semi-sphere with radius $\mathrm{R}=45 \mathrm{~nm}$, which was determined by 
scanning electron microscopy (Fig. S4). The wavelength of the AFM laser is $1300 \mathrm{~nm}$, which is far from the light absorption maxima of PTB7 [10]. All samples were prepared and stored inside a nitrogen filled glove-box and taken out just before AFM measurements. Local $I-V$ curves were corrected by subtraction of $I-V$ data obtained out of contact in order to compensate both for the current, which is proportional to $\mathrm{d} V / \mathrm{d} t$, and leakage current. Zero current correction was performed before each C-AFM measurement. All C-AFM measurements were executed by using the same probe in order to provide better reproducibility of the conductivity data, since nanoscale structure of the tips is unknown and may vary from one probe to the next. Each investigated area on the sample surface was measured only one time.
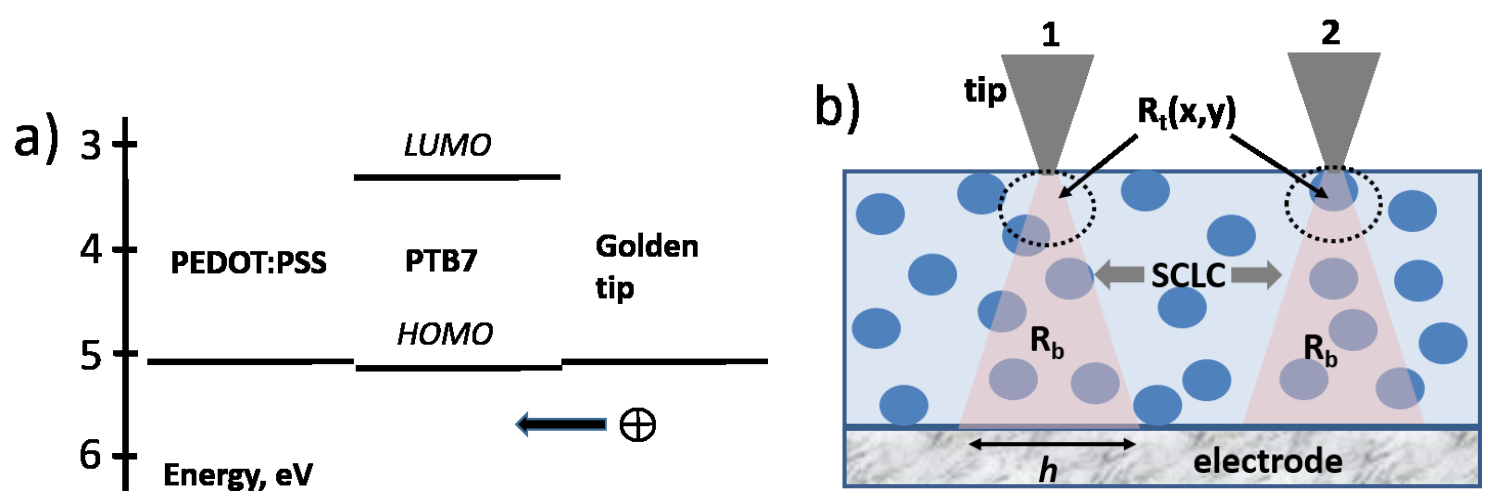

Fig. 2. a) Electronic structure of materials used: Fermi levels of the electrodes and HOMO/LUMO values for PTB7 [4]; b) scheme of C-AFM measurements of heterogeneous film.

Conventional SCLC measurements were performed on devices with the structure glass/ITO/PEDOT:PSS/PTB7/Au by using probe station Autolab. The hole mobility was calculated following the protocol published in [18]. Details on macroscopic SCLC data and C-AFM measurements are described in Supplementary Information. We assume ohmic contacts to the PTB7 in both C-AFM and conventional SCLC measurements. This assumption is based on electronic structure of the materials shown in Fig. 2a. The values of the electrodes work function and HOMO and LUMO of PTB7 were taken from literature $[4,11,18,27]$. Fermi level of electrodes may be slightly different when layers are contacting each other, e.g. Fermi level of gold can be lower than standard value 5.1-5.2 eV shown in Fig. 2a [18,28,29]. It is seen that difference between the HOMO of PTB7 and work functions of both electrodes is smaller than $0.2 \mathrm{eV}$, which confirms our assumption about presence of ohmic contacts for hole transport [30]. Additional support for ohmic contacts in the structure PEDOT:PSS/PTB7/Au is the successful use of conventional SCLC theory for hole mobility estimations in PTB7-based films by others [12]. Based on the abovementioned suggestions, we assume that bulk-limited current dominates in our measurements, which allows us to estimate charge carrier mobility in polymer film both locally and macroscopically.

\section{Results and discussion}

The current distribution measurements obtained by C-AFM in the neat PTB7 film (Fig. 3) reveal nonuniform conductivity with current features similar to that of PTB7:PCBM:DIO [10,11]. This observation indicates inhomogeneity of PTB7 organization. The electrical structure in neat PTB7 may be connected to existence of PTB7 nanocrystallites, which were previously detected by XRD [7-9]. Then the question arises: what is actually seen in current distribution image? Fig. $2 \mathrm{~b}$ demonstrates the scheme of C-AFM measurements of a heterogeneous PTB7 film, consisting of nanosized $\pi$-stacked crystals distributed inside the film. In each point of the scan the current is flowing between the tip and PEDOT:PSS through the volume, with a gradually increasing cross-section. This implies that the current signal at each point of Fig. $3 \mathrm{~b}$ is averaged over a volume, which is approximately a truncated cone with diameters of each end $d$ and $h$, where $d$ is tip-sample contact diameter and $h$ is diameter of current at the PEDOT:PSS interface (Fig. $2 b)$. 

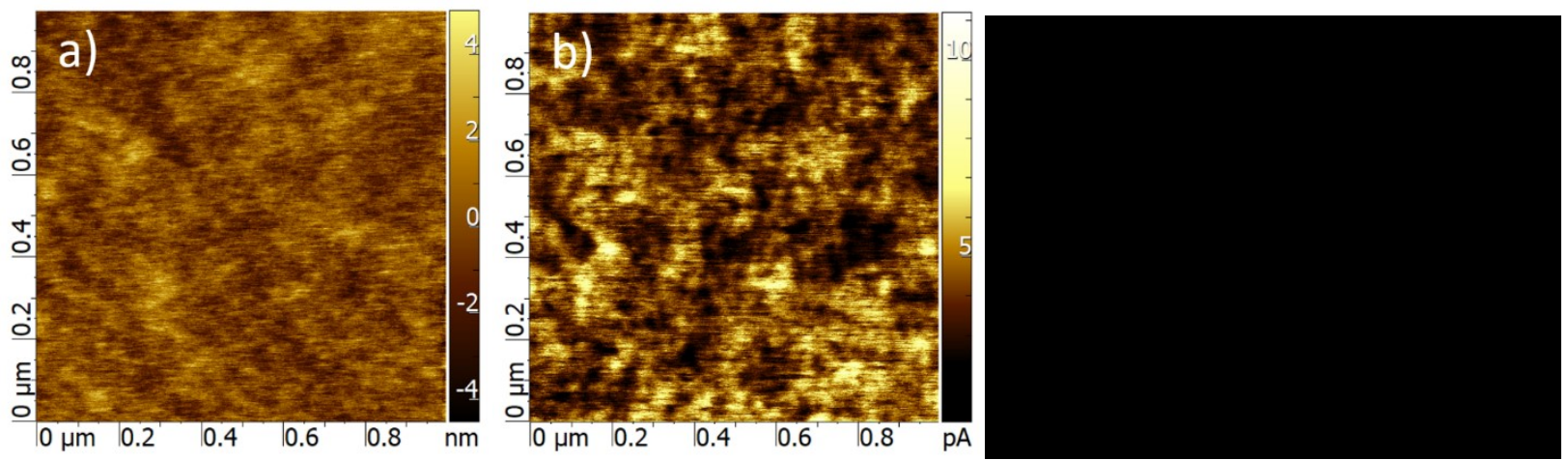

Fig. 3. Neat PTB7 film: a) topography, b) current distribution, c) cross-section of b) at $\mathrm{y}=0.6 \mu \mathrm{m}$.

In case of crystallites with sizes of few nanometers, approximately uniformly distributed within film volume, the measured current variations are mainly determined by the top part of the conical volume just under the tip, were sizes of crystallites are closer to the tip-sample contact diameter $d$ (Fig. 2b), which is typically in the range of 14-18 $\mathrm{nm}$ in our experiments as estimated by using Hertzian contact theory (see Supplementary Information). Total local resistance of the film can be written as a sum of the resistance of a thin top layer $R_{t}$ and resistance of the bottom part $R_{b}$. The latter one is nearly uniform when the size of inhomogeneities is much smaller than the analyzed volume. Then current variations in Figure $3 \mathrm{~b}$ are mainly determined by $R_{t}(x, y)$, i.e. by variations of the polymer structure just under the tip. Corresponding to a numerical simulation, the cross-section of current flow near the PEDOT:PSS electrode, $h$, can be as large as $h=120 \mathrm{~nm}$ for $100 \mathrm{~nm}$ thick film [22], which limits volume resolution of the method. The lateral resolution of the measurements is determined by tip-sample contact diameter $d$. It is important to note that current contrast in Figure $3 \mathrm{~b}$ has almost no correlation with topography image (Fig. 3a), which indicates nearly no influence of surface roughness on the measured current.

In order to perform a detailed study of the charge transport in the PTB7 film, an array of $25 I-V$ curves ( $5 \times 5$ square) was obtained on the surface of 8 separate PTB7 films of various thicknesses. It is important to determine the film thickness accurately, since current has a strong power dependence on it [18,22]. Here we use a method which is based on AFM measurements of the scratched sample. The scratched locations, consisting both PTB7 and PEDOT:PSS terraces, were measured and, by using statistical analysis, thickness of both films was determined (see Fig. 1). Such measurements were performed within $2 \mathrm{~mm}$ from the areas were current was measured in order to reduce influence of possible thickness variations over the film surface. The advantage of this method is that film thicknesses are determined for both PTB7 and PEDOT:PSS individually. The $25 \mathrm{I}-\mathrm{V}$ curves measured of the PTB7 film with a thickness of $102 \mathrm{~nm}$ are shown in Figs. 4a and 4b.

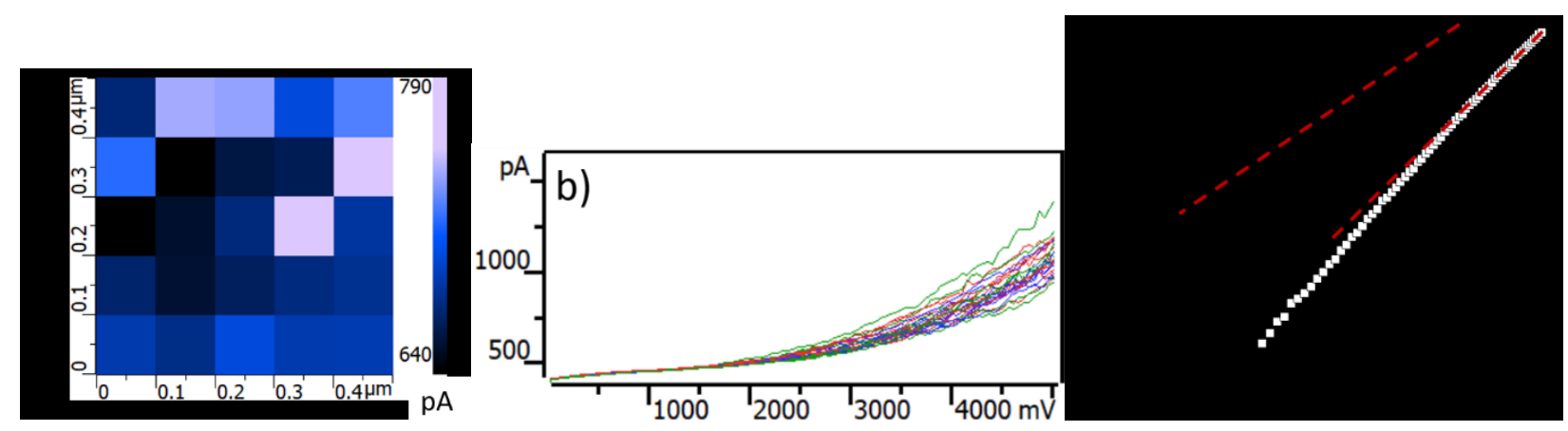

Fig. 4. a) Cross-section of array $5 \times 5 I-V$ curves at $3.5 \mathrm{~V}$, b) 25 local $I$ - $V$ curves, c) average local $I-V$ curve in double logarithmic scale without (1) and with $V_{b i}(2)$ taken into account.

The contrast in Fig. $3 \mathrm{~b}$ is formed by differences between $I-V$ curves in Fig. $4 \mathrm{~b}$ at voltages applied during measurements. The nonlinear character of $I-V$ curves is clearly seen. For further analysis, we will use part of the $I-V$ curves at positive voltage since such polarity provides higher current values (Fig. S1). Under such conditions the hole current through the polymer is observed, corresponding to energy levels of the 
materials used: the Fermi level of gold is close to the HOMO of PTB7 (Fig. 2a) [4,10-12]. At positive bias on the tip, the holes are injected from the tip into the polymer. For analysis of 8 samples with different thicknesses we use $I-V$ curves averaged over 25 curves for each sample. The average local $I-V$ curves are linearized with a double logarithmic scale and the slope of $I-V$ curves $\alpha_{i}$ above $0.5-1 \mathrm{~V}$ is typically between 2 and 3 or higher. Thus, we assume that in our measurements SCLC takes place at high voltages [14]. Further analysis is based on an algorithm described by Blacksley for macroscopic SCLC measurements [18] and adapted here for our C-AFM setup. First of all, the built-in voltage $V_{b i}$ was determined from average $I-V$ curves for each sample and then used in analysis. The estimated value $V_{b i}$ reduces the slope of the average local $I-V$ curves with a double logarithmic scale to pure quadratic with $\alpha_{r}=2.0 \pm 0.1$, which implies trap-free space charge limited current, as shown in Figure 4c. Fitting of standard macroscopic $I-V$ curves with the Murgatroyd equation [31] gives the field parameter $\gamma$ equal to 0 (see Supplementary Information), which confirms trap-free SCLC described by the Mott-Gurney law for our samples. The value of $V_{b i}$ estimated by using $\sqrt{I}-V$ plot was significantly higher for C-AFM results than for macroscopic SCLC data: in the range 1-2 V for C-AFM vs. $0.25 \mathrm{~V}$ for conventional measurements. Observed difference can be explained by an influence of the measuring conditions, first due to the AFM tip-sample interaction. An estimation of the local hole mobility from C-AFM measurements requires a modified Mott-Gurney equation, which was previously introduced by O. Reid et al. [22]. This approach is based on experimental $\mathrm{C}$-AFM data and numerical calculations. The main idea is to find the dependence of current density $J$ on sample thickness $L$ from experimental data and then modify the Murgatroyd equation for a C-AFM geometry. The proposed equation is written as [22]:

$$
I=A_{e f f} \alpha \varepsilon_{0} \varepsilon \mu \exp \left(0.89 \gamma\left(\frac{V}{L}\right)^{1 / 2}\right) \frac{V^{2}}{L^{3}} \delta\left(\frac{L}{d}\right)^{1.6},
$$

where $\alpha=8.2$ is the parameter from numerical calculations [22], $\varepsilon$ is the permittivity of material, $\mu$ is the hole mobility at zero field, $\gamma$ is the field dependence parameter of the mobility, $V$ is the tip-sample voltage, $L$ is the sample thickness, $d$ is the tip-sample contact diameter, $\delta=7.8$ is the scaling factor for fitting the CAFM mobility to the macroscopic data [22]. The model described in [22] was used by authors for calculations of the local mobility in various organic semiconductors, in particular polymer donors $\mathrm{P} 3 \mathrm{HT}$ and MDMO-PPV. Here, we use this approach to calculate and map local mobility in PTB7 films. First of all, the dependence of average current density on sample thickness $J-L$ was determined by C-AFM measurements of 8 samples of the neat PTB7 films with different thicknesses $\left(J=I / A_{\text {eff }}\right)$. The results of $J-L$ at $V_{t i p}=+2.9 \mathrm{~V}$ are shown in Figure 5a.
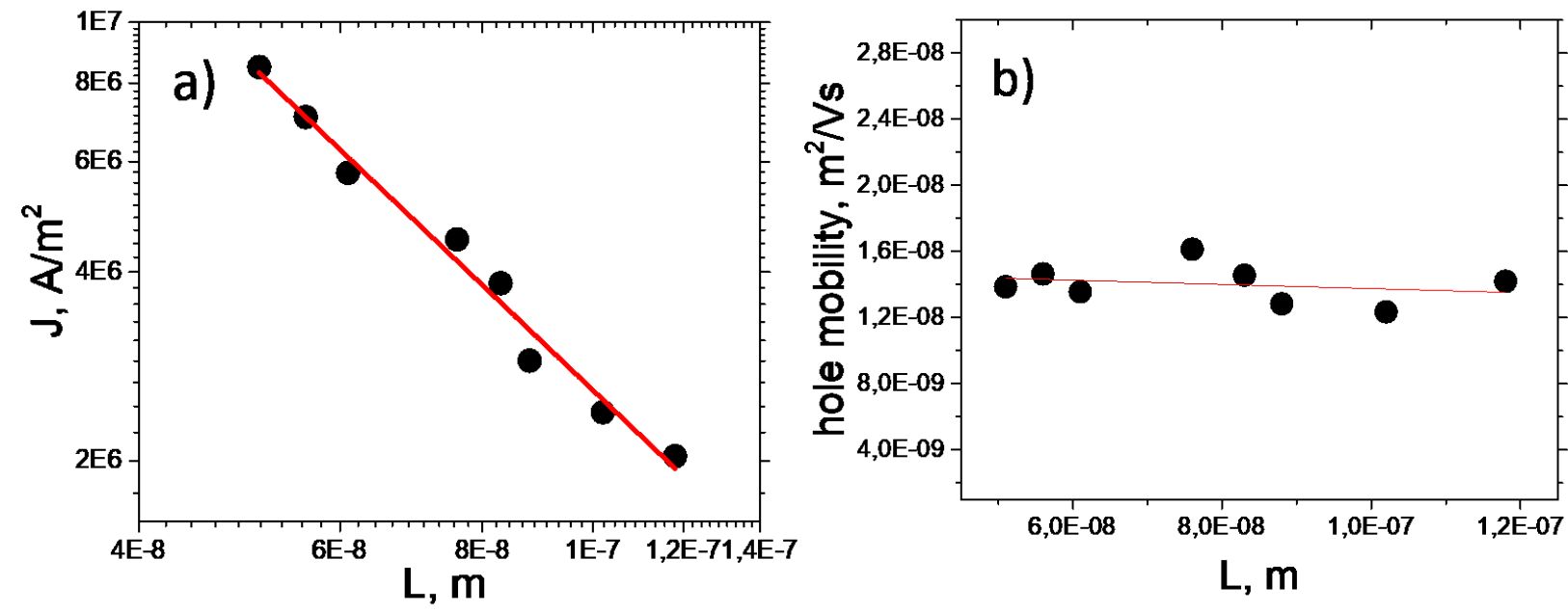

Fig. 5. Neat PTB7 films: a) $J-L$ dependence, b) average local hole mobility calculated for different thicknesses.

The linear character of dependence in the range of thicknesses used by us is clearly visible, which confirm results found before by O. Reid et al. [22]. However, the slope of the $J-L$ curve is larger in our results: $J \sim L^{-}$ ${ }^{1.7}$ vs $J \sim L^{-1.4}$ in [22]. This fact can be explained, first of all, by different type of probe with a larger size of 
the tip used in this work ( $\mathrm{R}=45 \mathrm{~nm}$ here and $40 \mathrm{~nm}$ in [22]) and, possibly, different tip geometry, determined by individual nanoscale features of each probe. The method of $V_{b i}$ estimation is also important, since this value has a drastic influence on the shape of local $I-V$ curves as shown in Fig. 4c. If $V_{b i}$ is not taken into account, then data in $J-L$ plot are scattered and no monotonic dependence occurs. Corresponding to standard theory, developed for geometry with flat electrodes, the $J \sim L^{-3}$ dependence should be expected if condition $L<<d$ is satisfied and, thus, standard a Mott-Gurney model is applicable [18,32]. For thick samples when $L>>d$, the hole injection from a point electrode takes place with $J \sim L^{-1}$ dependence [32], which was experimentally observed for $L>200 \mathrm{~nm}$ in [22]. The dependence $J-L$ is expected to also be shifted to lower $L$ values for a sharper tip due to the smaller contact diameter when similar conditions of measurements are used. It can also be expected that the power of $L$ is monotonically changing from -3 for thin samples to 1 for thick films. However, the accuracy of our data is not enough for the introduction of any nonlinearity to a $\log J-\log L$ plot and then the same power of $L-1.7$ should be used for whole range thicknesses. Similar results with a linear $\log J-\log L$ dependence in a wide range of thicknesses were described in [22]. In order to count $V_{b i}$ and a different slope of $\log J-\log L$, we rewrite equation (1) in the form:

$$
I=A_{e f f} \eta \varepsilon_{0} \varepsilon \mu \exp \left(0.89 \gamma\left(\frac{V-V_{b i}}{L}\right)^{1 / 2}\right) \frac{\left(V-V_{b i}\right)^{2}}{L^{3}}\left(\frac{L}{d}\right)^{3-\beta},
$$

where $\beta$ is the slope of $\log J-\log L$ (Fig. 5a), which, in general, is adjusted from 1 for thick films to 3 for very thin films and semi-empirical parameter $\eta=\alpha \delta$. The equation (2) was used for calculations of the hole mobility in the neat PTB7 films with different thicknesses (Fig. 5b). A relatively good agreement of mobility values for different thicknesses is observed, which is confirmed by very small slope of linear approximation of the data in Fig. 5b. The obtained value of C-AFM hole mobility $\mu=(1.40 \pm 0.12) \cdot 10^{-8} \mathrm{~m}^{2} \mathrm{~V}^{-}$ ${ }^{1} \mathrm{~s}^{-1}$ averaged for all thicknesses is close to the average macroscopic mobility $\mu_{0}=1.34 \cdot 10^{-8} \mathrm{~m}^{2} \mathrm{~V}^{-1} \mathrm{~s}^{-1}$ (see Supplementary Information). The difference between values of $\mu$ and $\mu_{0}$ is less than $10 \%$ and it may be result of errors in determinations of different parameters in model (2). Still, the values are close enough to each other to prove the ability of the model to estimate hole mobility in PTB7.

The next step is the application of this modified model for mapping of the hole mobility over a sample's surface. The same procedure of mobility calculations as described above was applied to a matrix of $5 \times 5 \mathrm{I}$ $V$ curves obtained on area $500 \times 500 \mathrm{~nm}$ for a sample with thickness of $102 \mathrm{~nm}$ (Fig. 4a). Distributions of built-in voltage and hole mobility over same surface area are shown in Fig. 6a and $6 \mathrm{~b}$ respectively. The image in Fig. $6 \mathrm{~b}$ was obtained by using equation (2) with values of $V_{b i}$ shown in Fig. 6 a.
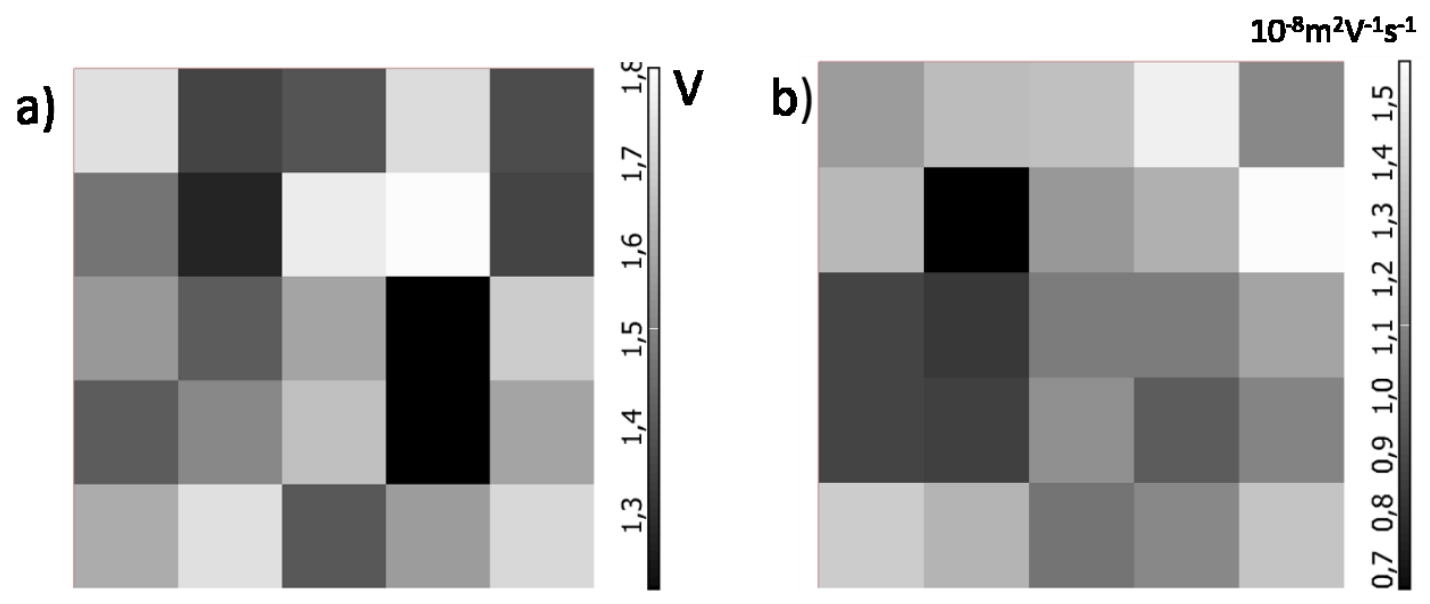

Fig. 6. Distributions of $V_{b i}$ (a) and hole mobility (b) on the same area as shown in Figure 4a. The total area of each plot is $0.5 \times 0.5$ microns.

The limited number of pixels is a result of slow standard technique of $I-V$ mapping, taking into account the 8 samples measured here. An increased number of pixels will make Fig. 4a look similar to Figure $2 \mathrm{~b}$. However, better resolution requires much longer acquisition time as well as an automated procedure for data treatment due to large data arrays. From the comparison of current distribution in Fig. $4 \mathrm{a}, V_{b i}$ and mobility maps in Fig. 6, it can be concluded that in general there is no direct correlation between all three 
images. Despite some features appearing similar, e.g. in the bottom right corner of $V_{b i}$ and mobility maps, decomposition of the $I-V$ map into $V_{b i}$ and mobility maps produces significantly different images. The average value of nanoscale mobility is $1.17 \times 10^{-8} \mathrm{~m}^{2} /(\mathrm{Vs})$, which is close to the macroscopic value of $1.34 \times 10^{-8} \mathrm{~m}^{2} /(\mathrm{Vs})$ obtained by regular SCLC on the same structure. At the same time, variations in mobility of more than 2 times, from 0.69 to $1.5910^{-8} \mathrm{~m}^{2} /(\mathrm{Vs})$ may be explained by some non-uniformity in the structure of PTB7 films, which was revealed earlier by XRD [7,8], and which is possibly observed in Figure 3b. The rms value of the hole mobility is $0.22 \times 10^{-8} \mathrm{~m}^{2} /(\mathrm{Vs})$, which is almost $20 \%$ from the average mobility. In the SCLC regime each pixel in Figures $4 \mathrm{a}$ and 6 represents the electrical properties inside a volume of the PTB7 film, which is an approximately truncated cone limited by the tip-sample contact diameter $d$ on top and diameter $h$ at PEDOT:PSS interface as it was discussed above. Thus, similar to discussion of current contrast, we suppose that variations of hole mobility in Fig. 6 reflect the ratio of ordered and amorphous PTB7 in the probed volume under the tip between $d$ and $h$.

The use of local SCLC measurements by C-AFM is a promising tool for nanoscale mobility measurements, however, further development and improvements of this technique are required. At the moment the main questions and sources of error of the method are: unknown $J-L$ dependences for an arbitrary AFM probe, significant influence of precision of film thickness measurements, and, a standard problem in AFM measurements, the unknown shape of tip and tip-sample contact area at the nanoscale. One of possible way to simplify local SCLC measurements is the use of probes with highly reproducible parameters, e.g. probes with spherical colloidal particles. It can also be expected that measurements of thick films with $J-L^{-1}$ dependence can significantly simplify the local mobility estimation, however, such thicknesses $>200 \mathrm{~nm}$ [22] are typically larger than the thicknesses of most successful OPV devices. In addition, there are still questions about large values of $V_{b i}$ in nanoscale $I-V$ measurements, obtained by same method as macroscopic values of $V_{b i}$. All abovementioned analysis was based on the suggestion that SCLC occurs in C-AFM results. This suggestion implies the existence of ohmic contacts to the film and confirmed by standard macroscopic measurements on the same structure as well as by the shape of the nanoscale $I-V$ curve. However, nanoscale conditions of measurements with strong probe-sample interaction in normal conditions may influence built-in voltages and be responsible for larger values of $V_{b i}$, which was determined from $I-V$ curves in the same way as the macroscopic value. Similar large values of $V_{b i}$ was observed by us on the neat MDMO-PPV $(\mathrm{HOMO}=5.2-5.4 \mathrm{eV})$ in a nitrogen atmosphere and thus the influence of humidity on $V_{b i}$ is expected to be minor. SCLC measurements on a PTB7 film performed in [4] with a relatively low work function $\mathrm{Al}$ film instead of Au electrode have also demonstrated a large value of $V_{b i}=1.5 \mathrm{~V}$. Thus, at this moment we suppose that modification of Au work function due to strong tip-sample interaction is responsible for an increased built-in voltage [28,29]. However, this conclusion requires additional experiments. Still, despite some questions, the method of mobility mapping described here allows for investigation of charge carrier mobility distribution over sample surface with nanoscale resolution and leads to reasonable agreement with macroscopic data.

\section{Conclusions}

In this work the nanoscale hole transport in PTB7 films was investigated by C-AFM. It was found that the current distribution is not uniform in a neat PTB7 film. The inhomogeneity of the current image was explained by the existence of ordered PTB7 crystallites. The presence of SCLC current was suggested and confirmed by local $I-V$ measurements. A procedure for local $I-V$ measurements at each point of the scan was performed and a modified semi-empirical equation was used to determine the nanoscale hole mobility. It was shown that the same procedure of built-in voltage estimation applied to macroscopic and nanoscale $I-V$ curves lead to significantly different values, which can be explained by strong tip-sample interaction in the AFM experiment. The two-dimensional array of $I-V$ curves was decomposed into two images with distributions of local built-in voltage and hole mobility by using a semi-empirical equation, which describes local $I-V$ dependences in the SCLC regime. Local mobility values on the surface of PTB7 films are influenced by the local structure and differ by more than two times and have rms value $0.22 \times 10^{-8} \mathrm{~m}^{2} /(\mathrm{Vs})$, which is almost $20 \%$ from the average mobility.

\section{Acknowledgements}


This work was supported by grant No. 19-12-00066 of the Russian Science Foundation. The authors thank D. Evplov (Horiba) and S. Leesment (NT-MDT SI) for their help with software adjustment and B. Ilyassov for sample preparation.

\section{References}

[1] L. Lu, T. Zheng, Q. Wu, A.M. Schneider, D. Zhao, L. Yu, Recent advances in bulk heterojunction polymer solar cells, Chem. Rev. 115 (2015) 12666-12731. https://doi.org/10.1021/acs.chemrev.5b00098.

[2] W. Zhao, S. Li, H. Yao, S. Zhang, Y. Zhang, B. Yang, J. Hou, Molecular optimization enables over $13 \%$ efficiency in organic solar cells, J. Am. Chem. Soc. 139 (2017) 7148-7151. https://doi.org/10.1021/jacs.7b02677.

[3] B. Fan, D. Zhang, M. Li, W. Zhong, Z. Zeng, L. Ying, F. Huang, Y. Cao, Achieving over 16\% efficiency for single-junction organic solar cells, Sci. China Chem. 62 (2019) 746-752. https://doi.org/10.1007/s11426-019-9457-5.

[4] Y. Liang, Z. Xu, J. Xia, S.-T. Tsai, Y. Wu, G. Li, C. Ray and L. Yu, For the bright future-bulk heterojunction polymer solar cells with power conversion efficiency of $7.4 \%$, Adv. Mater. 22 (2010) E135-E138. https://doi.org/10.1002/adma.200903528.

[5] Z. He, C. Zhong, S. Su, M. Xu, H. Wu and Y. Cao, Enhanced power-conversion efficiency in polymer solar cells using an inverted device structure, Nat. Photonics 6 (2012) 591-595. https://doi.org/10.1038/nphoton.2012.190.

[6] X. Ouyang, R. Peng, L. Ai, X. Zhang, Z. Ge, Efficient polymer solar cells employing a nonconjugated small-molecule electrolyte, Nat. Photon. 9 (2015) 520-524. https://doi.org/10.1038/nphoton.2015.126.

[7] M. R. Hammond, R. J. Kline, A. A. Herzing, L. J. Richter, D. S. Germack, H.-W. Ro, C. L. Soles, D. A. Fischer, T. Xu, L. Yu, M. F. Toney and D. M. DeLongchamp, Molecular order in highefficiency polymer/fullerene bulk heterojunction solar cells, ACS Nano 5 (2011) 8248-8257. https://doi.org/10.1021/nn202951e.

[8] B. A. Collins, Z. Li, J. R. Tumbleston, E. Gann, C. R. McNeill and H. Ade, Absolute measurement of domain composition and nanoscale size distribution explains performance in PTB7:PC71BM solar cells, Adv. Energy Mater. 3 (2012) 65-74. https://doi.org/10.1002/aenm.201200377.

[9] F. Liu, W. Zhao, J. R. Tumbleston, C. Wang, Y. Gu, D. Wang, A. L. Briseno, H. Ade and T. P. Russell, 2014. Understanding the Morphology of PTB7:PCBM Blends in Organic Photovoltaics. Adv. Energy Mater. 4, 1301377. https://doi.org/10.1002/aenm.201301377.

[10] G. J. Hedley, A. J. Ward, A. Alekseev, C. T. Howells, E. R. Martins, L. A. Serrano, G. Cooke, A. Ruseckas and I. D. W. Samuel, 2013. Determining the optimum morphology in highperformance polymer-fullerene organic photovoltaic cells. Nat. Commun. 4, 2867. https://doi.org/10.1038/ncomms3867.

[11] A Alekseev, GJ Hedley, A Al-Afeef, OA Ageev, IDW Samuel, Morphology and local electrical properties of ptb7: Pc71bm blends, Journal of Materials Chemistry A 3 (2015) 87068714. https://doi.org/10.1039/C5TA01224D.

[12] C.H. To, A. Ng, Q. Dong, A.B. Djurišić, J.A. Zapien, W.K. Chan, C. Surya, Effect of PTB7 properties on the performance of PTB7:PC71BM solar cells, ACS Appl. Mater. Interfaces 7 (2015) 13198-13207. https://doi.org/10.1021/am5085034.

[13] J.-H. Kim, J. Noh, H. Choi, J.-Y. Lee, T.-S. Kim, Mechanical properties of polymer-fullerene bulk heterojunction films: role of nanomorphology of composite films, Chem. Mater. 292017 3954-3961. https://doi.org/10.1021/acs.chemmater.7b00184.

[14] G. G. Malliaras, J. R. Salem, P. J. Brock, and C. Scott, Electrical characteristics and efficiency of single-layer organic light-emitting diodes, Phys. Rev. B 58 (1998) R13411-R13414. https://doi.org/10.1103/PhysRevB.58.R13411.

[15] P.W.M. Blom, M.C.J.M. Vissenberg, Charge transport in poly(p-phenylene vinylene) light-emitting diodes, Mat. Sci. Eng. R 27 (2000) 53-94. https://doi.org/10.1016/S0927796X(00)00009-7. 
[16] C. Goh, R.J. Kline, M.D. McGehee, E.N. Kadnikova, J.M.J. Fréchet, 2005. Molecularweight-dependent mobilities in regioregular Poly(3-hexyl-thiophene) diodes. Appl. Phys. Lett. 86, 122110. https://doi.org/10.1063/1.1891301.

[17] B. Ebenhoch, S.A.J. Thomson, K. Genevicius, G. Juška, I.D.W. Samuel, Charge carrier mobility of the organic photovoltaic materials PTB7 and PC71BM and its influence on device performance, Organic Electronics 22 (2015) 62-68. https://doi.org/10.1016/j.orgel.2015.03.013.

[18] J.C. Blakesley, F.A. Castro, W. Kylberg, G.F.A. Dibb, C. Arantes, R. Valaski, M. Cremona, J.S. Kim, J.-S. Kim, Towards reliable charge-mobility benchmark measurements for organic semiconductors, Organic Electronics $15 \quad$ (2014) 1263-1272. https://doi.org/10.1016/j.orgel.2014.02.008.

[19] A. Alexeev, J. Loos, M.M. Koetse, Nanoscale electrical characterization of semiconducting polymer blends by conductive atomic force microscopy (C-AFM), Ultramicroscopy 106 (2006) 191-199. https://doi.org/10.1016/j.ultramic.2005.07.003.

[20] D.C. Coffey, O.G. Reid, D.B. Rodovsky, G.P. Bartholomew, D.S. Ginger, Mapping local photocurrents in polymer/fullerene solar cells with photoconductive atomic force microscopy, Nano Letters 7 (2007) 738-744. https://doi.org/10.1021/n1062989e.

[21] X.-D. Dang, A. B. Tamayo, J. Seo, C. V. Hoven, B. Walker and T.-Q. Nguyen, Nanostructure and Optoelectronic Characterization of small molecule bulk heterojunction solar cells by photoconductive atomic force microscopy, Adv. Funct. Mater. 20 (2010) 3314-3321. https://doi.org/10.1002/adfm.201000799.

[22] O.G. Reid, K. Munechika, D.S. Ginger, Space charge limited current measurements on conjugated polymer films using conductive atomic force microscopy, Nano Lett. 8 (2008) 16021609. https://doi.org/10.1021/n10801551.

[23] X.-D. Dang, A. Mikhailovsky and T.-Q. Nguyen, 2010. Measurement of nanoscale external quantum efficiency of conjugated polymer:fullerene solar cells by photoconductive atomic force microscopy. Appl. Phys. Lett. 97, 113303. https://doi.org/10.1063/1.3483613.

[24] S. Cataldo, C. Sartorio, F. Giannazzo, A. Scandurra, B. Pignataro, Self-organization and nanostructural control in thin film heterojunctions, Nanoscale 6 (2014) 3566-3575. https://doi.org/10.1039/C3NR05027K.

[25] W. Hourani, K. Rahimi, I. Botiz, F.P.V. Koch, G. Reiter, P. Lienerth, T. Heiser, J.-L. Bubendorff, L. Simon, Anisotropic charge transport in large single crystals of p-conjugated organic molecules, Nanoscale 6 (2014) 4774-4780. https://doi.org/10.1039/C3NR05858A.

[26] J.E. Sader, J.W.M. Chon, P. Mulvaney, Calibration of rectangular atomic force microscope cantilevers, Rev. Sci. Instr. 70 (1999) 3967-3969. https://doi.org/10.1063/1.1150021.

[27] A.M. Nardes, M. Kemerink, M.M. de Kok, E. Vinken, K. Maturova, R.A.J. Janssen, Conductivity, work function, and environmental stability of PEDOT:PSS thin films treated with sorbitol, Org. Electron. 9 (2008) 727-734. https://doi.org/10.1016/j.orgel.2008.05.006.

[28] I. Lange, J.C. Blakesley, J. Frisch, A. Vollmer, N. Koch, D. Neher, 2011. Band Bending in Conjugated Polymer Layers. Phis. Rev. Lett. 106, 216402. https://doi.org/10.1103/PhysRevLett.106.216402.

[29] W. Osikowicz, M.P. de Jong, S. Braun, Energetics at Au top and bottom contacts on conjugated polymers, Appl. Phys. Lett. 88 (2006) 193504. https://doi.org/10.1063/1.2201627.

[30] W. Mönch, Ohmic contacts, in: Electronic Properties of Semiconductor Interfaces, Springer Series in Surface Sciences, vol 43, Springer, Berlin, Heidelberg, 2004, pp. 227-230. https://doi.org/10.1007/978-3-662-06945-5 11.

[31] P. N. Murgatroyd, Theory of space-charge-limited current enhanced by Frenkel effect, J. Phys. D: Appl. Phys. 3 (1970) 151-156. https://doi.org/10.1088/0022-3727/3/2/308.

[32] M.A. Lampert, P. Mark, Current Injection in Solids, Academic Press, New York, 1970 


\section{Supplementary Information}

\section{Macroscopic mobility measurements of PTB7 films by SCLC.}

Structure glass/ITO/PEDOT:PSS/PTB7 film/Au was used for SCLC measurements. PTB7 film was spincoated from solution in chlorobenzene at different rotation speed of substrate. Au electrodes were thermally evaporated with an active area of device of $8 \mathrm{~mm}^{2}$. The unified protocol of mobility measurements and calculations was used [S1]. The results of SCLC measurements are shown below:

Table 1. SCLC measurements of the neat PTB7 films.

\begin{tabular}{|l|l|l|l|l|l|}
\hline Sample & $V_{b i}, \mathrm{~V}$ & $\mu_{0}, \mathrm{~m}^{2} /(\mathrm{Vs})$ & $\gamma,(\mathrm{m} / \mathrm{V})^{0.5}$ & $L, \mathrm{~nm}$ & $\log I-\log U$ slope \\
\hline PTB7 (neat) $1000 \mathrm{rpm}$ & 0.25 & $1.34 * 10^{-8}$ & 0 & 172 & 1.94 \\
\hline PTB7 (neat) 2000 rpm & 0.25 & $1.33 * 10^{-8}$ & 0 & 108 & 2.00 \\
\hline
\end{tabular}

\section{Protocol of SCLC measurements by C-AFM.}

2.1. Samples for C-AFM were produced by same method as devices in part 1 but without top Au electrode. The raw I-V curves are shown in Fig. S1. Strong asymmetry between dependences at positive and negative biases are clearly seen, indicating more efficient hole injection from AFM probe.

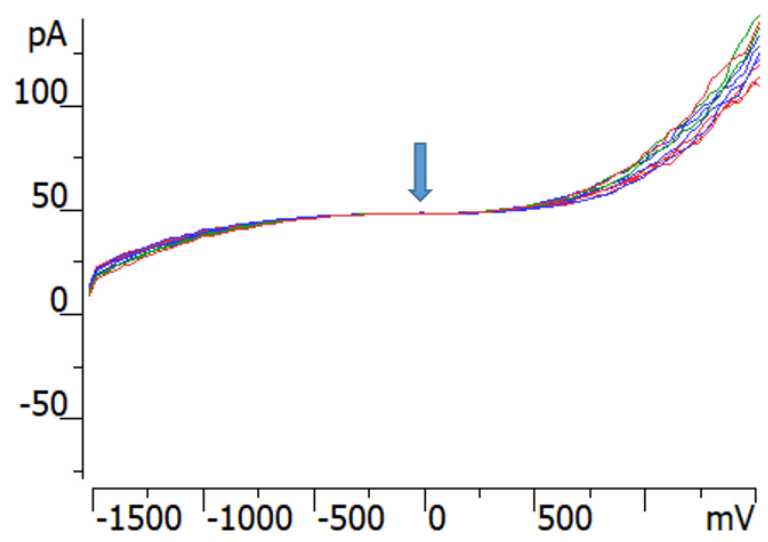

Fig. S1. PTB7 film: 10 raw $I-V$ curves. The arrow indicates zero point.

2.2. The gold-coated probe CSG10/Au (NT-MDT) was biased during C-AFM measurements and sample was grounded. Positive voltage on probe was applied, which implies hole injection from tip to the film. The $I-V$ measurements for SCLC were performed point-by-point at square area by using contact probe. It is important that same probe is used for all samples, which are selected for comparison, since tip size and tip shape are individual parameters, which cannot be controlled. In order to avoid currents caused by leakage and voltage changes $\mathrm{d} V / \mathrm{d} t$, the measurements of "zero" $I-V$ out of contact were performed and then these data were subtracted from initial curve (Fig. S2). 


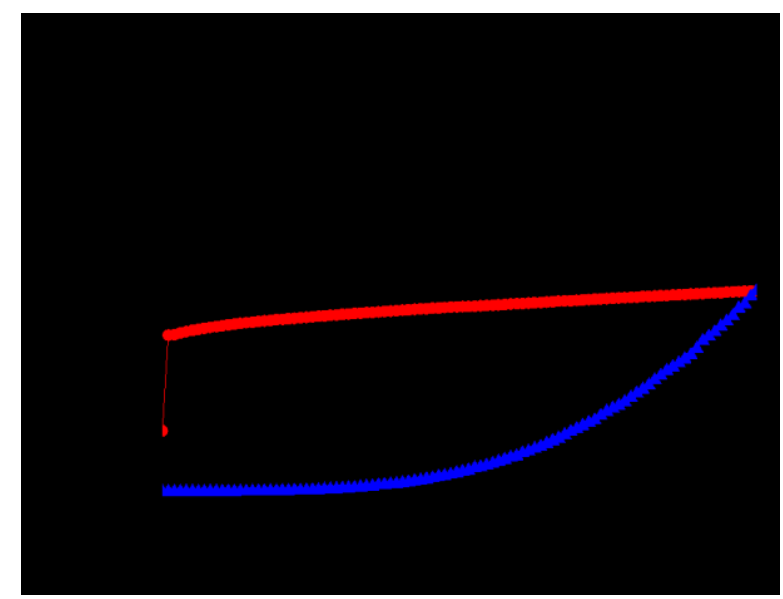

Fig. S2. $I-V$ measurements on PTB7 film: 1 - raw $I-V$ data; 2 - "zero" $I-V$ curve; 3 - $I-V$ curve (1) after subtraction curve (2).

2.3. Tip-sample force during C-AFM measurements was minimized in order to reduce possible surface damage. Total tip-sample force is calculated from force-distance curve $(F-z)$ as a sum of adhesion force and force caused by lever deflection $F=F_{c}+F_{a d}$ (Fig. S3). Forces were calculated as $F=k D F L$, where $k=0.28$ $\mathrm{N} / \mathrm{m}$ is cantilever force constant determined by Sader method and cantilever deflection $D F L$ is determined from force-distance curve assuming 45 degree slope in contact region, as shown in Fig. S3a. Tip-sample force was estimated for each set of $I-V$ measurements and then used for calculation of the contact area for each experiment.

\section{Force $F(z)$}

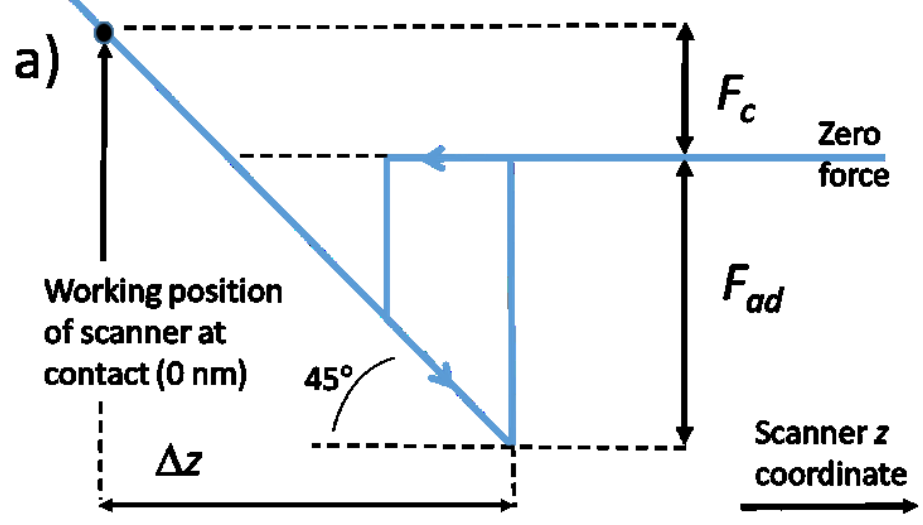

\section{Deflection $D F L$, a.u.}

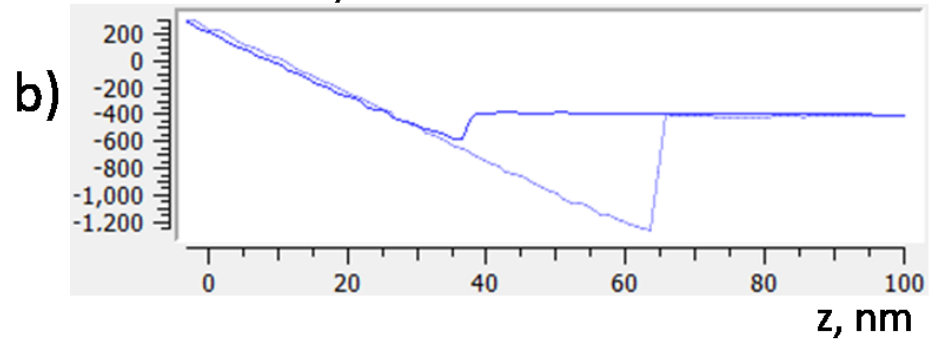

Fig. S3. Force-distance curve: a) scheme of calculation of total tip-sample force; b) experimental deflection $(z)$ curve obtained by CSC10/Au probe on the neat PTB7 film.

2.4. The tip-sample contact diameter was determined by using Hertzian contact theory for contact of sphere with plain surface: $d=2 \sqrt[3]{\frac{3 F R}{4 E^{*}}}$, where reduced Young's modulus $E^{*}$ is determined from equation $\frac{1}{E^{*}}=$ $\frac{1-v_{t}^{2}}{E_{t}}+\frac{1-v_{s}^{2}}{E_{S}}, R$ is tip radius determined from SEM image ( $R \sim 45 \mathrm{~nm}$ for our probe, see Fig. S4), E is Young's 
modulus, $v$ is Poisson's ratio, $t$ and $s$ denote tip and sample respectively. Young's modulus of PTB7-based films were taken from recent work [S2]: $E_{s}=0.44 \mathrm{GPa}$ for neat PTB7. Poisson's ratio $v=0.3$ was used, since no data on this value was found in literature. Same parameters for gold were $78 \mathrm{GPa}$ and 0.44, correspondingly. The calculated effective contact area $A_{\text {eff }}$ was used for calculations of current density $J=I / A_{\text {eff. }}$. Standard values of tip-sample force were in the range $5-10 \mathrm{nN}$ and tip-sample contact diameter $d$ was between $14-18 \mathrm{~nm}$.

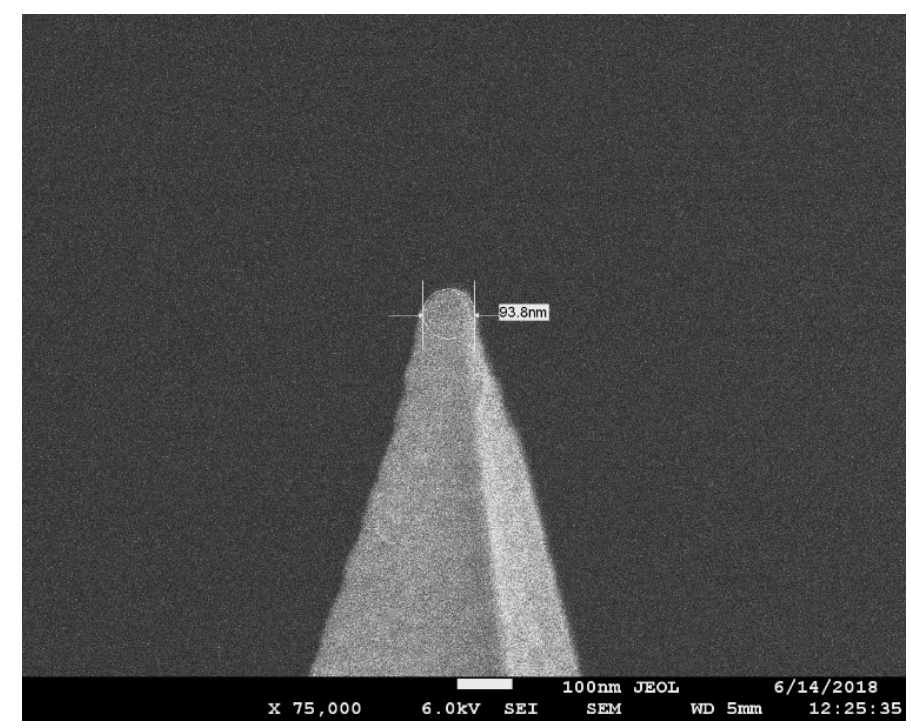

Fig. S4. SEM image of the CSG10/Au tip used in measurements.

2.5. Next steps of local mobility calculations correspond to standard SCLC procedure [18]. The built-in voltage $V_{b i}$ is determined for average local $I-V$ curve from $\sqrt{I-V}$ plot. Then equation (2) from main text is used for fitting experimental data. This procedure was also used for analysis of individual $I-V$ curves.

\section{References}

[S1] J.C. Blakesley, F.A. Castro, W. Kylberg, G.F.A. Dibb, C. Arantes, R. Valaski, M. Cremona, J.S. Kim, J.-S. Kim, Towards reliable charge-mobility benchmark measurements for organic $\begin{array}{lllll}\text { semiconductors, } & \text { Organic } & \text { Electronics } & 15 & \text { (2014) }\end{array}$ https://doi.org/10.1016/j.orgel.2014.02.008.

[S2] J.-H. Kim, J. Noh, H. Choi, J.-Y. Lee, T.-S. Kim, Mechanical properties of polymer-fullerene bulk heterojunction films: role of nanomorphology of composite films, Chem. Mater. 292017 3954-3961. https://doi.org/10.1021/acs.chemmater.7b00184. 